\title{
Nanotechnology and Drones, „Boom-Art” in the Advanced Tech
}

\author{
Gabriela OPAIT ${ }^{\star}$
}

\begin{tabular}{l}
\hline \multicolumn{1}{c}{ A R T I C L E I N F O } \\
\hline Article history: \\
Accepted March 2020 \\
Available online May 2020 \\
\hline JEL Classification \\
C1, C12, C2 \\
Keywords: \\
Nanoparticles, Nanocrystals, \\
Nanosensors, Nanomedicine, \\
Nanoelectronics, Nanowhiskers, 3D \\
printed sensors, Nanoscale \\
Spectroscopy, Star Wars X-Wing \\
Starfighter drone \\
\hline
\end{tabular}

\section{A B S T R A C T}

The nanotechnology and the drones represent two zones of great interest in many countries which have as aim only one vector, namely the advance to high quotas of the science. The purposes of this scientific approach follow to present for the lovers of the nanotechnology and for the passionates of drones, a „smart display" which offer them the informations about the immediate future concerning the worldwide market values of the nanotechnology, respectively the worldwide consumptions and the worldwide revenues as effects of the marketing of drones.

\section{Introduction}

The nanotechnology managed to display as through the symbiosis of the science with the research, it „sculpts” art. The shapes of the nanotechnology have as „scoreline” the productions of devices and materials at atomic and molecular scale. New performances in nanotechnology are amazing: nanostructured rubber which cans to substitute the human tissue, heart attack supervised on chip, nanoscale device which cans to produce giant power terahertz concerning the waves, fascinating magnets with sounds, nanoscale magnetic vortex which produces chaos, device which parrots the brain cells utilized in the process concerning the human vision, 3D printed sensors which can to generate a breath test in the diabetic zone of the population or nanomaterials which can be used in the process of localization for the early cancer. In the first level of this original approach, we can „savour" the arsenal of techniques which estimates the market value of the worldwide nanotechnology in 2020. In the second step, we make acquaintance with the „protocol” which describes the identification of the worldwide consumption of drones, in 2020. In the third step, we meet the „technology" which serves at the selection value concerning the worldwide revenue obtained through the marketing of drones, in the same year. For to shape these targets we ticked and used the method the prognosis which was positioned in the value by the „Least Squares Method”. The patent of innovation concerning „Least Squares Method” is achieved in 1823, by Johann Carl Friedrich Gauss. Through this instrument, we have the possibility to "touch" the „physiognomies” which belong to the equations's parameters and at the same time, we can to make previsions of the phenomenons focused in our researchs.

2. The mathematical design which reflects the estimation in 2020 concerning the market value of the worldwide nanotechnology

Table 1. The ranking regarding the worldwide market values regarding the nanotechnology, in 2010-2019

\begin{tabular}{|c|c|}
\hline YEARS & $\begin{array}{c}\text { THE WORLDWIDE MARKET VALUES CONCERNING } \\
\text { THE NANOTECHNOLOGY } \\
\text { (billions \$) } \\
\left(\xi_{i}\right)\end{array}$ \\
\hline 2010 & 15,7 \\
\hline 2011 & 20,1 \\
\hline 2012 & 20,7 \\
\hline 2013 & 22,9 \\
\hline 2014 & 26,0 \\
\hline
\end{tabular}




\begin{tabular}{|l|l|}
\hline 2015 & 27,0 \\
\hline 2016 & 36,2 \\
\hline 2017 & 48,9 \\
\hline 2018 & 56,3 \\
\hline 2019 & 64,2 \\
\hline
\end{tabular}

Source: „Statista Portal the United States of America”

- if the "portfolio of the mathematical operations” for the $\xi$ variable, where $\xi=$ the market values of the worldwide nanotechnology, provides a linear itinerary $\xi_{t_{i}}=a+b \cdot t_{i}, a$ and $b$ will be [5]:

$$
a=\frac{\left|\begin{array}{ll}
\sum_{i=1}^{n} \xi_{i} & \sum_{i=1}^{n} t_{i} \\
\sum_{i=1}^{n} \xi_{i} t_{i} & \sum_{i=1}^{n} t_{i}{ }^{2}
\end{array}\right|}{\left|\begin{array}{cc}
n & \sum_{i=1}^{n} t_{i} \\
\sum_{i=1}^{n} t_{i} & \sum_{i=1}^{n} t_{i}{ }^{2}
\end{array}\right|}=\frac{\sum_{i=1}^{n} \xi_{i} \sum_{i=1}^{n} t_{i}{ }^{2}-\sum_{i=1}^{n} \xi_{i} t_{i} \sum_{i=1}^{n} t_{i}}{n \sum_{i=1}^{n} t_{i}{ }^{2}-\left(\sum_{i=1}^{n} t_{i}\right)^{2}} \quad b=\frac{\left|\begin{array}{cc}
n & \sum_{i=1}^{n} \xi_{i} \\
\sum_{i=1}^{n} t_{i} & \sum_{i=1}^{n} \xi_{i} t_{i}
\end{array}\right|}{\left|\begin{array}{cc}
n & \sum_{i=1}^{n} t_{i} \\
\sum_{i=1}^{n} t_{i} & \sum_{i=1}^{n} t_{i}{ }^{2}
\end{array}\right|}=\frac{n \sum_{i=1}^{n} \xi_{i} t_{i}-\sum_{i=1}^{n} t_{i} \sum_{i=1}^{n} \xi_{i}}{n \sum_{i=1}^{n} t_{i}{ }^{2}-\left(\sum_{i=1}^{n} t_{i}\right)^{2}}
$$

Table 2. The rank regarding the worldwide market values of the nanotechnology, if this brings forward a linear itinerary

\begin{tabular}{|c|c|c|c|c|c|c|}
\hline \multirow{2}{*}{ YEARS } & \multirow{2}{*}{$\begin{array}{c}\text { THE WORLDWIDE } \\
\text { MARKET VALUES } \\
\text { CONCERNING THE } \\
\text { NANOTECHNOLOGY } \\
\text { (billions \$) } \\
\left(\xi_{i}\right)\end{array}$} & \multicolumn{5}{|c|}{ LINEAR TENDENCY } \\
\hline & & $t_{i}$ & $t_{i}^{2}$ & $t_{i} \xi_{i}$ & $\xi_{t_{i}}=a+b t_{i}$ & $\mid \xi_{i}-\xi_{t_{i}}$ \\
\hline 2010 & 15,7 & -5 & 25 & $-78,5$ & 11,09545455 & 4,6 \\
\hline 2011 & 20,1 & -4 & 16 & $-80,4$ & 15,63636364 & 4,5 \\
\hline 2012 & 20,7 & -3 & 9 & $-62,1$ & 20,17727273 & 0,5 \\
\hline 2013 & 22,9 & -2 & 4 & $-45,8$ & 24,71818182 & 1,8 \\
\hline 2014 & 26,0 & -1 & 1 & $-26,0$ & 29,25909091 & 3,3 \\
\hline 2015 & 27,0 & +1 & 1 & $+27,0$ & 38,34090909 & 11,3 \\
\hline 2016 & 36,2 & +2 & 4 & $+72,4$ & 42,88181818 & 6,7 \\
\hline 2017 & 48,9 & +3 & 9 & $+146,7$ & 47,42272727 & 1,5 \\
\hline 2018 & 56,3 & +4 & 16 & $+225,2$ & 51,96363636 & 4,3 \\
\hline 2019 & 64,2 & +5 & 25 & $+321,0$ & 56,50454546 & 7,7 \\
\hline TOTAL & 338 & & 110 & 499,5 & 338 & 46,2 \\
\hline
\end{tabular}

$$
\begin{gathered}
a=\frac{338 \cdot 110-499,5 \cdot 0}{10 \cdot 110-0^{2}}=33,8 \\
b=\frac{10 \cdot 499,5-0 \cdot 338}{10 \cdot 110-0^{2}}=4,540909091 \\
v_{I}=\left[\frac{\sum_{i=1}^{m}\left|\xi_{i}-\xi_{t_{i}}^{I}\right|}{n}: \frac{\sum_{i=1}^{m} \xi_{i}}{n}\right] \cdot 100=\frac{\sum_{i=1}^{m}\left|\xi_{i}-\xi_{t_{i}}^{I}\right|}{\sum_{i=1}^{m} \xi_{i}} \cdot 100=\frac{46,2}{338} \cdot 100=13,67 \%
\end{gathered}
$$

- if the „portfolio of the mathematical operations” for $\xi$ variable, where $\xi=$ the market values of the worldwide nanotechnology, provides a parabolic itinerary $\xi_{t_{i}}=a+b \cdot t_{i}+c t_{i}^{2}, a$ and $b$ will be [5]: 
Table 3. The rank concerning the worlwide market values of the nanotechnology, if this brings forward a quadratic itinerary

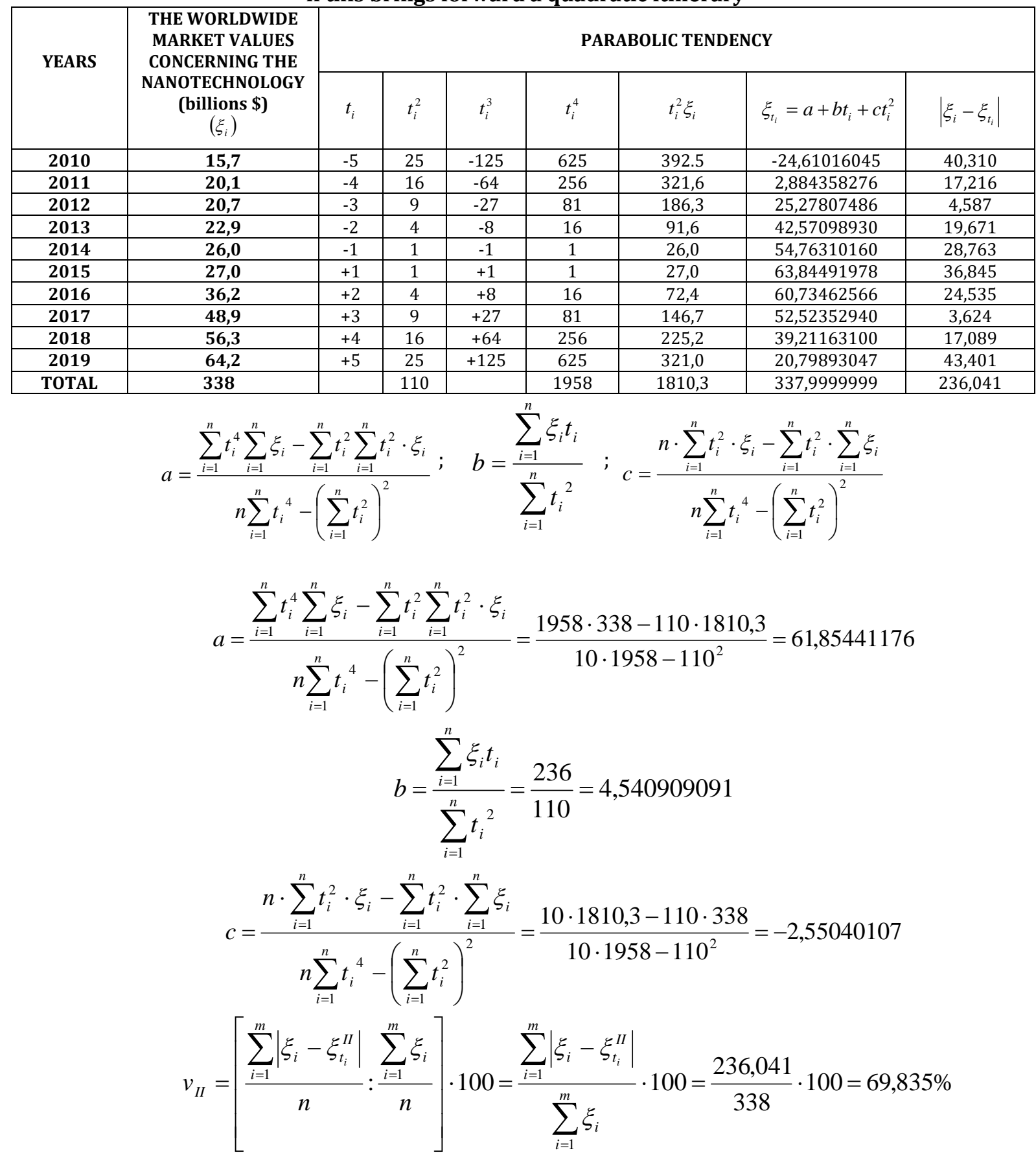

- if the "portfolio of the mathematical operations" for $\xi$ variable, where $\xi=$ the market values of the worldwide nanotechnology, provides a parabolic itinerary of three degree $\xi_{t_{i}}=a+b \cdot t_{i}+c t_{i}^{2}+d t_{i}^{3}, a, b$, $c$ and $d$ will be [5]: 
Table 4 The rank regarding the worldwide market values of the nanotechnology, if this brings forward a quadratic itinerary of three degree

\begin{tabular}{|c|c|c|c|c|c|c|c|c|c|}
\hline \multirow{2}{*}{ YEARS } & \multirow{2}{*}{$\begin{array}{c}\text { THE WORLDWIDE } \\
\text { MARKET VALUES } \\
\text { CONCERNING THE } \\
\text { NANOTECHNOLOGY } \\
\text { (billions \$) } \\
\left(\xi_{i}\right)\end{array}$} & \multicolumn{8}{|c|}{ PARABOLIC TENDENCY OF THREE DEGREE } \\
\hline & & $t_{i}$ & $t_{i}^{2}$ & $t_{i}^{3}$ & $t_{i}^{4}$ & $t_{i}^{6}$ & $t_{i}^{3} \xi_{i}$ & $\xi_{t_{i}}=a+b \cdot t_{i}+c t_{i}^{2}+d t_{i}^{3}$ & $\left|\xi_{i}-\xi_{t_{i}}\right|$ \\
\hline 2010 & 15,7 & -5 & 25 & -125 & 625 & 15625 & $-1962,5$ & $-26,69057993$ & 42,391 \\
\hline 2011 & 20,1 & -4 & 16 & -64 & 256 & 4096 & $-1286,4$ & 3,300442244 & 16,800 \\
\hline 2012 & 20,7 & -3 & 9 & -27 & 81 & 729 & $-558,9$ & 26,80371591 & 6,104 \\
\hline 2013 & 22,9 & -2 & 4 & -8 & 16 & 64 & $-183,2$ & 44,16597765 & 21,266 \\
\hline 2014 & 26,0 & -1 & 1 & -1 & 1 & 1 & -26 & 55,73396407 & 29,734 \\
\hline 2015 & 27,0 & +1 & 1 & +1 & 1 & 1 & 27 & 62,87405731 & 35,874 \\
\hline 2016 & 36,2 & +2 & 4 & +8 & 16 & 64 & 289,6 & 59,13963731 & 22,940 \\
\hline 2017 & 48,9 & +3 & 9 & +27 & 81 & 729 & 1320,3 & 50,99788836 & 2,098 \\
\hline 2018 & 56,3 & +4 & 16 & +64 & 256 & 4096 & 3603,2 & 38,79554704 & 17,504 \\
\hline 2019 & 64,2 & +5 & 25 & +125 & 625 & 15625 & 8025,0 & 22,87934995 & 41,321 \\
\hline TOTAL & 338 & & 110 & & 1958 & 41030 & 9248,1 & 337,9999999 & 236,032 \\
\hline
\end{tabular}

$$
\begin{aligned}
& a=\frac{\sum_{i=1}^{n} t_{i}^{4} \sum_{i=1}^{n} \xi_{i}-\sum_{i=1}^{n} t_{i}^{2} \sum_{i=1}^{n} t_{i}^{2} \cdot \xi_{i}}{n \sum_{i=1}^{n} t_{i}{ }^{4}-\left(\sum_{i=1}^{n} t_{i}^{2}\right)^{2}}=\frac{1958 \cdot 338-110 \cdot 1810,3}{10 \cdot 1958-110^{2}}=61,85441176 \\
& b=\frac{\sum_{i=1}^{n} t_{i}^{6} \cdot \sum_{i=1}^{n} t_{i} \cdot \xi_{i}-\sum_{i=1}^{n} t_{i}^{4} \cdot \sum_{i=1}^{n} t_{i}^{3} \cdot \xi_{i}}{\sum_{i=1}^{n} t_{i}{ }^{2} \cdot \sum_{i=1}^{n} t_{i}^{6}-\left(\sum_{i=1}^{n} t_{i}^{4}\right)^{2}}=\frac{41030 \cdot 499,5-1958 \cdot 9248,1}{110 \cdot 41030-1958^{2}}=3,512257187 \\
& c=\frac{n \cdot \sum_{i=1}^{n} t_{i}^{2} \cdot \xi_{i}-\sum_{i=1}^{n} t_{i}^{2} \cdot \sum_{i=1}^{n} \xi_{i}}{n \sum_{i=1}^{n} t_{i}^{4}-\left(\sum_{i=1}^{n} t_{i}^{2}\right)^{2}}=\frac{10 \cdot 1810,3-110 \cdot 3718}{10 \cdot 1958-110^{2}}=-2,55040107 \\
& d=\frac{\sum_{i=1}^{n} t_{i}^{2} \cdot \sum_{i=1}^{n} t_{i}^{3} \cdot \xi_{i}-\sum_{i=1}^{n} t_{i}^{4} \cdot \sum_{i=1}^{n} t_{i} \cdot \xi_{i}}{\sum_{i=1}^{n} t_{i}^{2} \cdot \sum_{i=1}^{n} t_{i}^{6}-\left(\sum_{i=1}^{n} t_{i}^{4}\right)^{2}}=\frac{110 \cdot 9248,1-1958 \cdot 499,5}{110 \cdot 41030-1958^{2}}=0,057789432 \\
& V_{I I I}=\left[\frac{\sum_{i=1}^{m}\left|\xi_{i}-\xi_{t_{i}}^{I I I}\right|}{n}: \frac{\sum_{i=1}^{m} \xi_{i}}{n}\right] \cdot 100=\frac{\sum_{i=1}^{m}\left|\xi_{i}-\xi_{t_{i}}^{I I I}\right|}{\sum_{i=1}^{m} \xi_{i}} \cdot 100=\frac{236,032}{338} \cdot 100=69,832 \%
\end{aligned}
$$

- if the "portfolio of the mathematical operations" for $\xi$ variable, where $\xi=$ the market values of the worldwide nanotechnology, provides an exponential itinerary $\xi_{t_{i}}=a b^{t_{i}}$, a and $b$ will be [5]:

$$
\lg a=\frac{\left|\begin{array}{ll}
\sum_{i=1}^{n} \lg \xi_{i} & \sum_{i=1}^{n} t_{i} \\
\sum_{i=1}^{n} t_{i} \lg \xi_{i} & \sum_{i=1}^{i=1} t_{i}{ }^{2}
\end{array}\right|}{\left|\begin{array}{cc}
n & \sum_{i=1}^{n} t_{i} \\
\mid \sum_{i=1}^{n} t_{i} & \sum_{i=1}^{n} t_{i}{ }^{2}
\end{array}\right|}=\frac{\sum_{i=1}^{n} \lg \xi_{i} \sum_{i=1}^{n} t_{i}{ }^{2}-\sum_{i=1}^{n} t_{i} \lg \xi_{i} \sum_{i=1}^{n} t_{i}}{n \sum_{i=1}^{n} t_{i}{ }^{2}-\left(\sum_{i=1}^{n} t_{i}\right)^{2}} \quad \lg b=\frac{\left|\begin{array}{cc}
n & \sum_{i=1}^{n} \lg \xi_{i} \\
\sum_{i=1}^{n} t_{i} & \sum_{i=1}^{n} t \\
i & \lg \xi_{i}
\end{array}\right|}{\left|\begin{array}{cc}
n & \sum_{i=1}^{n} t_{i} \\
\sum_{i=1}^{n} t_{i} & \sum_{i=1}^{n} t_{i}{ }^{2}
\end{array}\right|}=\frac{n \sum_{i=1}^{n} t_{i} \lg \xi_{i}-\sum_{i=1}^{n} \lg \xi_{i} \sum_{i=1}^{n} t_{i}}{n \sum_{i=1}^{n} t_{i}{ }^{2}-\left(\sum_{i=1}^{n} t_{i}\right)^{2}}
$$


Table 5 The rank concerning the worldwide market values of the nanotechnology, if this brings forward an exponential itinerary

\begin{tabular}{|c|c|c|c|c|c|c|}
\hline \multirow{2}{*}{ YEARS } & \multirow{2}{*}{$\begin{array}{c}\text { THE WORLDWIDE } \\
\text { MARKET VALUES } \\
\text { CONCERNING THE } \\
\text { NANOTECHNOLOGY } \\
\text { (billions \$) } \\
\left(\xi_{i}\right)\end{array}$} & \multicolumn{5}{|c|}{ EXPONENTIAL TENDENCY } \\
\hline & & $\lg \xi_{i}$ & $t_{i} \lg \xi_{i}$ & $\lg \xi_{t_{i}}=\lg a+t_{i} \lg b$ & $\xi_{t_{i}}=a b^{t_{i}}$ & $\left|\xi_{i}-\xi_{t_{i}}\right|$ \\
\hline 2010 & 15,7 & 1,195899652 & $-5,979498262$ & 1,192665821 & 15,58325617 & 0,117 \\
\hline 2011 & 20,1 & 1,303196057 & $-5,212784230$ & 1,250672558 & 17,81035426 & 2,290 \\
\hline 2012 & 20,7 & 1,315970345 & $-3,947911036$ & 1,308686906 & 20,35574051 & 0,344 \\
\hline 2013 & 22,9 & 1,359835482 & $-2,719670965$ & 1,366701254 & 23,26490342 & 0,365 \\
\hline 2014 & 26,0 & 1,414973348 & $-1,414973348$ & 1,424715602 & 26,58983253 & 0,590 \\
\hline 2015 & 27,0 & 1,431363764 & $+1,431363764$ & 1,540744298 & 34,73316009 & 7,733 \\
\hline 2016 & 36,2 & 1,558708571 & $+3,117417141$ & 1,598758646 & 39,69708763 & 3,497 \\
\hline 2017 & 48,9 & 1,689308859 & $+5,067926577$ & 1,656772994 & 45,37044031 & 3,530 \\
\hline 2018 & 56,3 & 1,750508395 & $+7,002033579$ & 1,714787342 & 51,85460639 & 4,445 \\
\hline 2019 & 64,2 & 1,807535028 & $+9,037675140$ & 1,772801690 & 59,26546415 & 4,935 \\
\hline TOTAL & 338 & 14,82729950 & 6,381578360 & & & 27,846 \\
\hline
\end{tabular}

$$
\begin{gathered}
\lg a=\frac{14,8272995 \cdot 110-6,38157836 \cdot 0}{10 \cdot 110-0^{2}}=1,48272995 \\
\lg b=\frac{10 \cdot 6,38157836-14,8272995 \cdot 0}{10 \cdot 110-0^{2}}=0,058014348 \\
V_{\exp }=\left[\frac{\sum_{i=1}^{n}\left|\xi_{i}-\xi_{t_{i}}^{\exp }\right|}{n}: \frac{\sum_{i=1}^{n} \xi_{i}}{n}\right] \cdot 100=\frac{\sum_{i=1}^{n}\left|\xi_{i}-\xi_{t_{i}}^{\exp }\right|}{\sum_{i=1}^{n} \xi_{i}} \cdot 100=\frac{27,846}{338} \cdot 100=8,24 \% \\
V_{\exp }=8,24 \%<v_{I}=13,67 \%<v_{I I I}=69,832 \%<v_{I I}=69,835 \%
\end{gathered}
$$

The „portfolio of the mathematical operations” which focuses the worldwide market values concerning the Nanotechnology, brings forward an exponential route $\xi_{t_{i}}=a b^{t_{i}}$

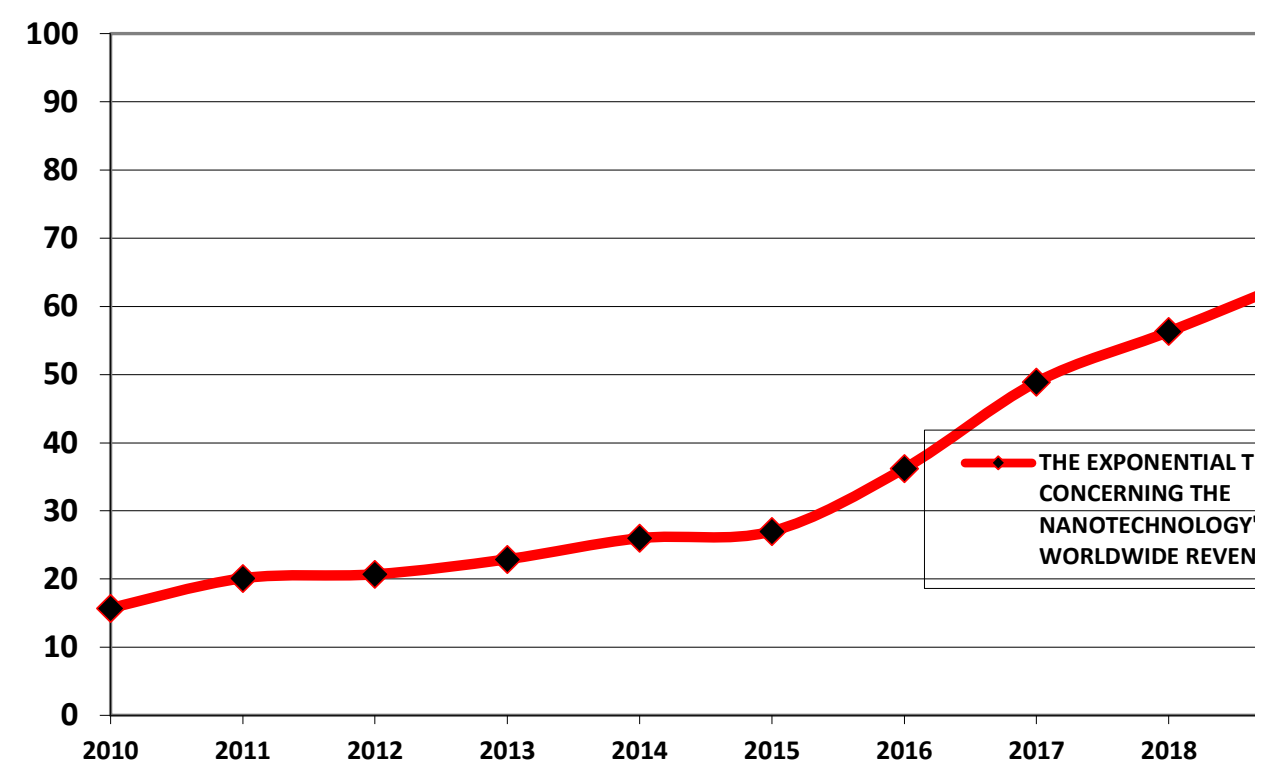

Graph 1 The exponential itinerary for the worldwide market values which stipulate the dynamics of the Nanotechnoly's worldwide revenues

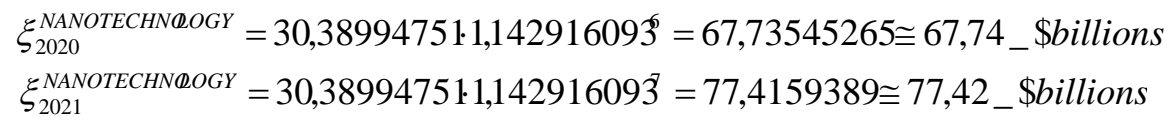


3. The mathematical design which exhibits the estimation in 2020 regarding the worldwide consumption of drones

Table 6 The ranking regarding the worldwide consumption of drones,

in 2015-2019

\begin{tabular}{|c|c|}
\hline YEARS & $\begin{array}{c}\text { THE WORLDWIDE CONSUMPTION OF DRONES } \\
\text { (thousands) } \\
\left(\omega_{i}\right)\end{array}$ \\
\hline 2015 & 6403 \\
\hline 2016 & 10244 \\
\hline 2017 & 15878 \\
\hline 2018 & 23818 \\
\hline 2019 & Source: „Statista Portal the United States of America” \\
\hline
\end{tabular}

- if the „portfolio of the mathematical operations” for $\omega$ variable, where $\omega=$ the worldwide consumption of drones, provides a linear itinerary $\omega_{t_{i}}=a+b \cdot t_{i}, a$ and $b$ will be [5]:

Table 7 The rank of numbers concerning the worldwide consumption of drones, if this brings forward a linear itinerary

\begin{tabular}{|c|c|c|c|c|c|c|}
\hline \multirow{2}{*}{ YEARS } & \multirow{2}{*}{$\begin{array}{c}\text { THE WORLDWIDE } \\
\text { MARKET OF DRONES } \\
\text { (thousands) } \\
\left(\omega_{i}\right)\end{array}$} & \multicolumn{5}{|c|}{ LINEAR TENDENCY } \\
\hline & & $t_{i}$ & $t_{i}^{2}$ & $t_{i} \omega_{i}$ & $\omega_{t_{i}}=a+b t_{i}$ & $\omega_{i}-\omega_{t_{i}}$ \\
\hline 2015 & 6403 & -2 & 4 & -12806 & 4207,8 & 2195 \\
\hline 2016 & 10244 & -1 & 1 & -10244 & 11191,8 & 948 \\
\hline 2017 & 15878 & 0 & 0 & 0 & 18175,8 & 2298 \\
\hline 2018 & 23818 & +1 & 1 & 23818 & 25159,8 & 1342 \\
\hline 2019 & 34536 & +2 & 4 & 69072 & 32143,8 & 2392 \\
\hline TOTAL & 90879 & 0 & 10 & 69840 & 90879 & 9175 \\
\hline
\end{tabular}

$$
\begin{gathered}
a=\frac{\sum_{i=1}^{n} \omega_{i} \sum_{i=1}^{n} t_{i}{ }^{2}-\sum_{i=1}^{n} \omega_{i} t_{i} \sum_{i=1}^{n} t_{i}}{n \sum_{i=1}^{n} t_{i}{ }^{2}-\left(\sum_{i=1}^{n} t_{i}\right)^{2}}=\frac{90879 \cdot 10}{5 \cdot 10}=18175,8 \quad b=\frac{n \sum_{i=1}^{n} \omega_{i} t_{i}-\sum_{i=1}^{n} t_{i} \sum_{i=1}^{n} \omega_{i}}{n \sum_{i=1}^{n} t_{i}{ }^{2}-\left(\sum_{i=1}^{n} t_{i}\right)^{2}}=\frac{5 \cdot 69840}{5 \cdot 10}=6984 \\
v_{I}=\left[\frac{\sum_{i=1}^{n}\left|\omega_{i}-\omega_{t_{i}}^{I}\right|}{n}: \frac{\sum_{i=1}^{n} \omega_{i}}{n}\right] \cdot 100=\frac{\sum_{i=1}^{n}\left|\omega_{i}-\omega_{t_{i}}^{I}\right|}{\sum_{i=1}^{n} \omega_{i}} \cdot 100=\frac{9175}{90879} \cdot 100=10,10 \%
\end{gathered}
$$

- if the „portfolio of the mathematical operations” for $\omega$ variable, where $\omega=$ the worldwide consumption of drones, provides a quadratic itinerary $\omega_{t_{i}}=a+b \cdot t_{i}+c t_{i}^{2}, a$ and $b$ will be [5]:

\begin{tabular}{|c|c|c|c|c|c|c|c|}
\hline \multicolumn{8}{|c|}{ if this brings forward a quadratic itinerary } \\
\hline \multirow{2}{*}{ YEARS } & \multirow{2}{*}{$\begin{array}{c}\text { THE WORLDWIDE } \\
\text { MARKET OF } \\
\text { DRONES } \\
\text { (thousands) } \\
\left(\omega_{i}\right)\end{array}$} & \multicolumn{6}{|c|}{ PARABOLIC TENDENCY } \\
\hline & & $t_{i}$ & $t_{i}^{2}$ & $t_{i}^{4}$ & $t_{i}^{2} \omega_{i}$ & $\omega_{t_{i}}=a+b t_{i}+c t_{i}^{2}$ & $\left|\omega_{i}-\omega_{t_{i}}\right|$ \\
\hline 2015 & 6403 & -2 & 4 & 16 & 25612 & 6502,085718 & 99 \\
\hline 2016 & 10244 & -1 & 1 & 1 & 10244 & 10044,65715 & 199 \\
\hline 2017 & 15878 & 0 & 0 & 0 & 0 & 15881,51429 & 4 \\
\hline 2018 & 23818 & +1 & $\frac{0}{1}$ & 1 & 23818 & 24012,65715 & 195 \\
\hline 2019 & 34536 & +2 & 4 & 16 & 138144 & 34438,08572 & 98 \\
\hline TOTAL & 90879 & 0 & 10 & 34 & 197818 & 90879 & 595 \\
\hline
\end{tabular}

Table 8 The rank of numbers regarding the worldwide consumption of drones, 


$$
\begin{gathered}
a=\frac{\sum_{i=1}^{n} t_{i}^{4} \sum_{i=1}^{n} \omega_{i}-\sum_{i=1}^{n} t_{i}^{2} \sum_{i=1}^{n} t_{i}^{2} \cdot \omega_{i}}{n \sum_{i=1}^{n} t_{i}{ }^{4}-\left(\sum_{i=1}^{n} t_{i}^{2}\right)^{2}}=\frac{34 \cdot 90879-10 \cdot 197818}{5 \cdot 34-10^{2}}=15881,51429 \quad b=\frac{\sum_{i=1}^{n} \omega_{i} t_{i}}{\sum_{i=1}^{n} t_{i}{ }^{2}}=\frac{69840}{10}=6984 \\
c=\frac{n \cdot \sum_{i=1}^{n} t_{i}^{2} \cdot \omega_{i}-\sum_{i=1}^{n} t_{i}^{2} \cdot \sum_{i=1}^{n} \omega_{i}}{n \sum_{i=1}^{n} t_{i}{ }^{4}-\left(\sum_{i=1}^{n} t_{i}^{2}\right)^{2}}=\frac{5 \cdot 197818-10 \cdot 90879}{5 \cdot 34-10^{2}}=1147,142857 \\
v_{I I}=\left[\frac{\sum_{i=1}^{n}\left|\omega_{i}-\omega_{t_{i}}^{I I}\right|}{n}: \frac{\sum_{i=1}^{n} \omega_{i}}{n}\right] \cdot 100=\frac{\sum_{i=1}^{n}\left|\omega_{i}-\omega_{t_{i}}^{I I}\right|}{\sum_{i=1}^{n} \omega_{i}} \cdot 100=\frac{595}{90879} \cdot 100=0,66 \%
\end{gathered}
$$

- if the „portfolio of the mathematical operations” for $\omega$ variable, where $\omega=$ the worldwide consumption of drones, provides a parabolic itinerary of three degree $\omega_{t_{i}}=a+b \cdot t_{i}+c t_{i}^{2}+d t_{i}^{3}, a, b, c$ and $d$ will be [5]:

Table 9 The rank of numbers concerning the worldwide consumption of drones, if this brings forward a quadratic itinerary of three degree

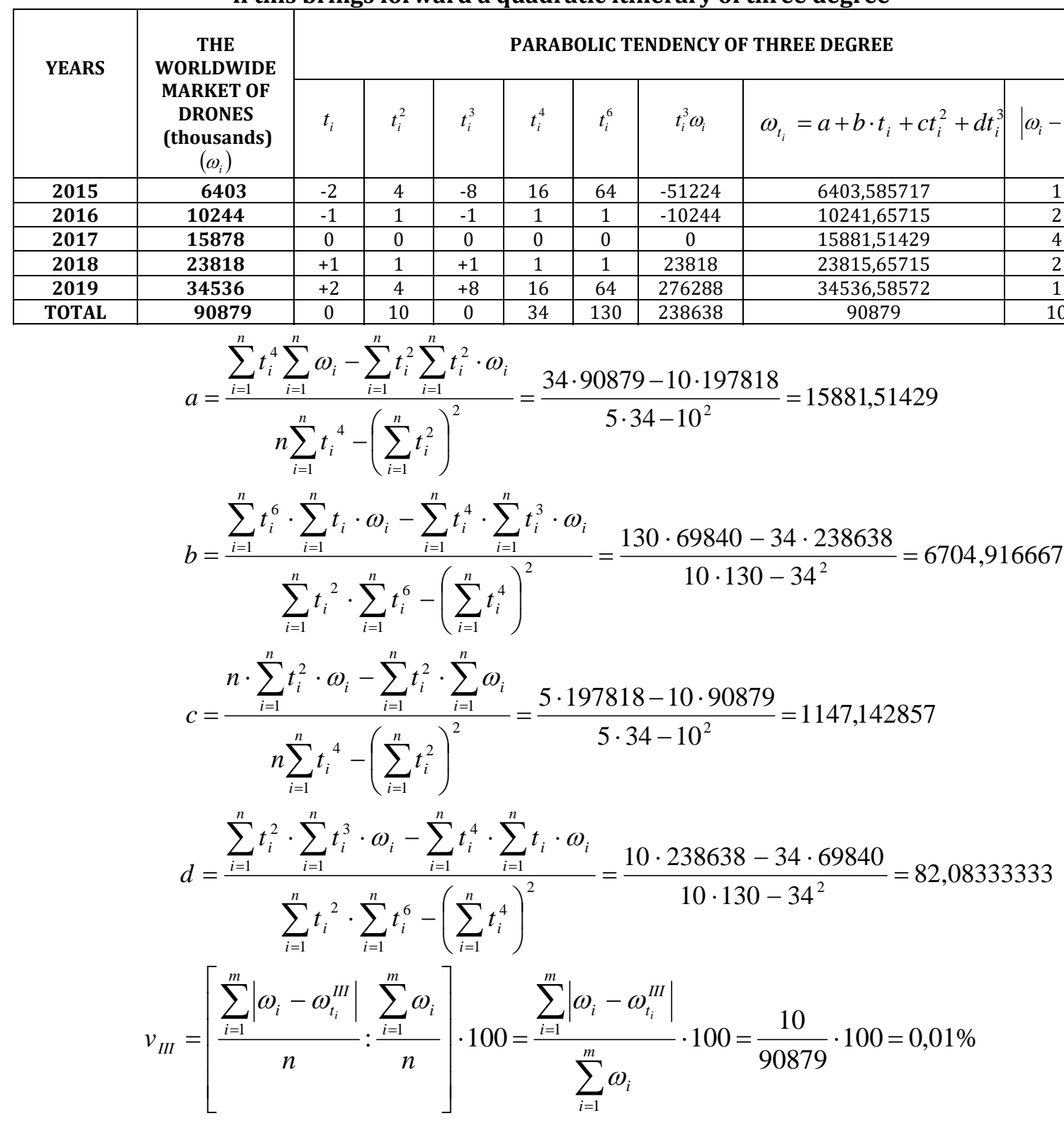


- if the „portfolio of the mathematical operations” for $\omega$ variable, where $\omega=$ the worldwide consumption of drones, provides an exponential itinerary $\omega_{t_{i}}=a b^{t_{i}}, a$ and $b$ will be [5]:

Table 10 The rank of numbers regarding the worldwide consumption of drones, if this brings forward an exponential itinerary

\begin{tabular}{|c|c|c|c|c|c|c|c|}
\hline & \multirow{2}{*}{$\begin{array}{c}\text { THE WORLDWIDE } \\
\text { YEARS }\end{array}$} & \multicolumn{9}{|c|}{$\begin{array}{c}\text { EXPONENTIAL TENDENCY } \\
\text { DRONES } \\
\end{array}$} & $\begin{array}{c}\text { (thousands) } \\
\left(\omega_{i}\right)\end{array}$ & $t_{i}$ & $\lg \omega_{i}$ & $t_{i} \lg \omega_{i}$ & $\lg \omega_{t_{i}}=\lg a+t_{i} \lg b$ & $\omega_{t_{i}}=a b^{t_{i}}$ & $\left|\omega_{i}-\omega_{t_{i}}\right|$ \\
\cline { 3 - 9 } & $\mathbf{6 4 0 3}$ & -2 & 3,806383502 & $-7,612767004$ & 3,820522683 & 6614,890859 & 212 \\
\hline $\mathbf{2 0 1 5}$ & $\mathbf{1 0 2 4 4}$ & -1 & 4,010469570 & $-4,010469570$ & 4,003543961 & 10081,93658 & 162 \\
\hline $\mathbf{2 0 1 6}$ & $\mathbf{1 5 8 7 8}$ & 0 & 4,200795798 & 0 & 4,186565239 & 15366,15604 & 512 \\
\hline $\mathbf{2 0 1 7}$ & $\mathbf{2 3 8 1 8}$ & +1 & 4,376905291 & 4,376905291 & 4,369586517 & 15366,15604 & 398 \\
\hline $\mathbf{2 0 1 8}$ & $\mathbf{3 4 5 3 6}$ & +2 & 4,538272036 & 9,076544071 & 4,552607795 & 35695,03359 & 1159 \\
\hline $\mathbf{2 0 1 9}$ & $\mathbf{9 0 8 7 9}$ & 0 & 20,9328262 & 1,830212788 & & & 2443 \\
\hline TOTAL & & &
\end{tabular}

$$
\begin{gathered}
\lg a=\frac{\sum_{i=1}^{n} \lg \omega_{i} \sum_{i=1}^{n} t_{i}^{2}-\sum_{i=1}^{n} t_{i} \lg \omega_{i} \sum_{i=1}^{n} t_{i}}{n \sum_{i=1}^{n} t_{i}^{2}-\left(\sum_{i=1}^{n} t_{i}\right)^{2}}=\frac{20,9328262 \cdot 10}{5 \cdot 10}=4,186565239 \\
\lg b=\frac{n \cdot \sum_{i=1}^{n} t_{i} \lg \omega_{i}-\sum_{i=1}^{n} \lg \omega_{i} \sum_{i=1}^{n} t_{i}}{n \sum_{i=1}^{n} t_{i}^{2}-\left(\sum_{i=1}^{n} t_{i}\right)^{2}}=\frac{5 \cdot 1,830212788}{5 \cdot 10}=0,183021278 \\
v_{\exp }=\left[\frac{\sum_{i=1}^{n}\left|\omega_{i}-\omega_{t_{i}}^{\exp }\right| \sum_{i=1}^{n} \omega_{i}}{n}\right] \cdot 100=\frac{\sum_{i=1}^{n}\left|\omega_{i}-\omega_{t_{i}}^{\exp }\right|}{\sum_{i=1}^{n} \omega_{i}} \cdot 100=\frac{2443}{90879} \cdot 100=2,69 \% \\
v_{I I I}=0,01 \%<v_{I I}=0,66 \%<v_{\exp }=2,69 \%<v_{I}=10,10 \%
\end{gathered}
$$

The „portfolio of the mathematical operations” which supervisas the worldwide consumption of drones, brings forward a quadratic itinerary of three degree $\omega_{t_{i}}=a+b \cdot t_{i}+c t_{i}^{2}+d t_{i}^{3}$

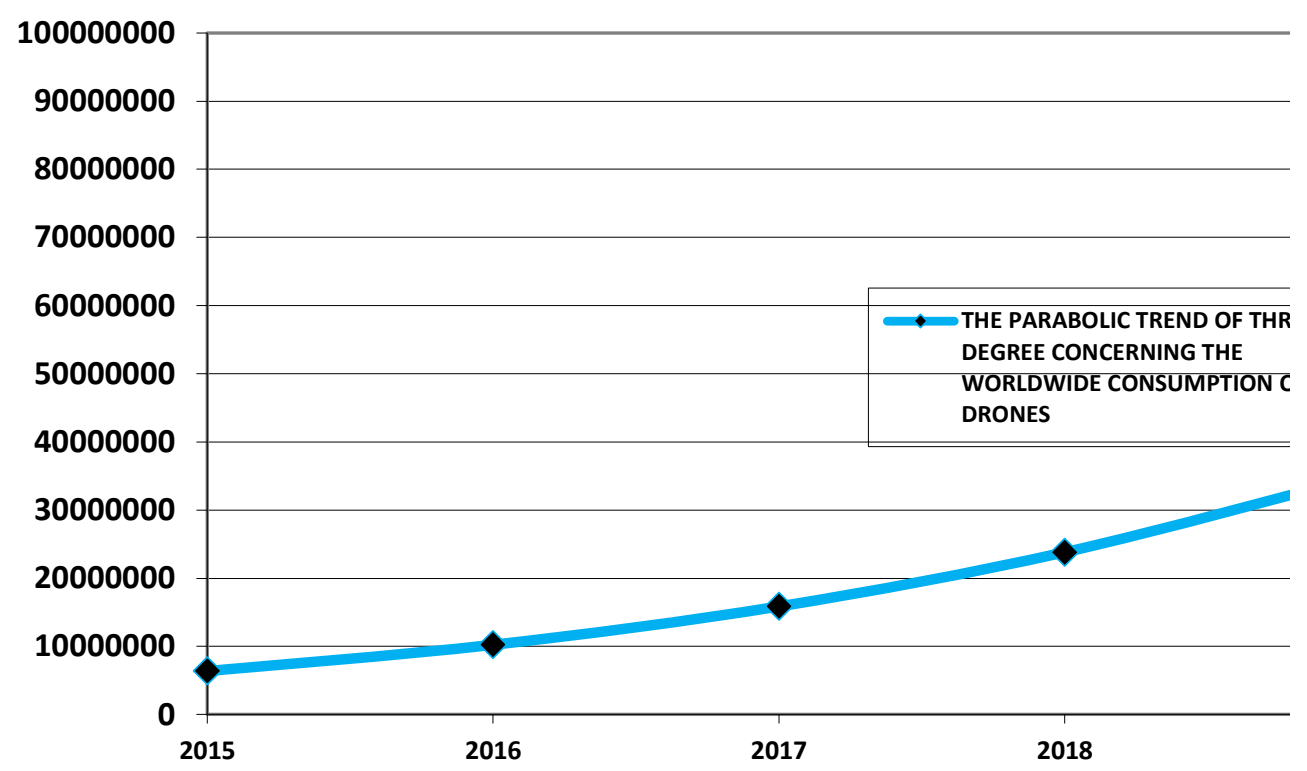

Graph 2 The quadratic itineray of three degree for the values which stipulate the evolution of the worldwide consumption of drones 
$\omega_{2020}^{\text {WORLDWIDE_DRONES }}=15881,51429+6704,916667 \cdot 3+1147,142857 \cdot 3^{2}+82,08333333 \cdot 3^{3} \cong 48537 \cdot 10^{3} \cong 49 \_$millions_drones $\omega_{2021}^{\text {WORLDWIDE_DRONES }}=15881,51429+6704,916667 \cdot 4+1147,142857 \cdot 4^{2}+82,08333333 \cdot 4^{3} \cong 66309 \cdot 10^{3} \cong 66 \_$millions_drones

4. The mathematical design which unveils the estimation in 2020 concerning the worldwide revenues obtained through the marketing of drones

Table 6 The ranking for the worlwide revenues concerning the merchandising of drones, in 2015-2019

\begin{tabular}{|c|c|}
\hline YEARS & $\begin{array}{c}\text { THE WORLDWIDE REVENUES REGARDING THE MARKETING OF DRONES } \\
\text { (millions \$) } \\
\left(\wp_{i}\right)\end{array}$ \\
\hline 2015 & $\mathbf{1 8 6 5 , 6 4}$ \\
\hline 2016 & 2385,29 \\
\hline 2017 & 2950,90 \\
\hline 2018 & 3528,73 \\
\hline 2019 & $\mathbf{4 0 7 4 , 3 1}$ \\
\hline
\end{tabular}

Source: „Statista Portal the United States of America

- if the "portfolio of the mathematical operations" for $\omega$ variable, where $\wp=$ the worlwide revenues concerning the marketing of drones, provides a linear itinerary $\wp_{t_{i}}=a+b \cdot t_{i}, a$ and $b$ will be [5]:

Table 7 The rank of numbers concerning the worlwide revenues concerning the merchandising of drones, if this brings forward a linear itinerary

\begin{tabular}{|c|c|c|c|c|c|c|}
\hline \multirow{2}{*}{ YEARS } & $\begin{array}{c}\text { THE WORLDWIDE } \\
\text { REVENUES } \\
\text { CONCERNING } \\
\text { THE DRONES } \\
\text { (millions \$) } \\
\left(\wp_{i}\right)\end{array}$ & $t_{i}$ & $t_{i}^{2}$ & $t_{i} \wp_{i}$ & $\wp_{t_{i}}=a+b t_{i}$ & $\wp_{i}-\wp_{t_{i}} \mid$ \\
\cline { 3 - 8 } & $\mathbf{1 8 6 5 , 6 4}$ & -2 & 4 & $-3731,28$ & 1848,822 & 16,818 \\
\hline $\mathbf{2 0 1 5}$ & $\mathbf{2 3 8 5 , 2 9}$ & -1 & 1 & $-2385,29$ & 2404,898 & 19,608 \\
\hline $\mathbf{2 0 1 6}$ & $\mathbf{2 9 5 0 , 9 0}$ & 0 & 0 & 0 & 2960,974 & 10,074 \\
\hline $\mathbf{2 0 1 7}$ & $\mathbf{3 5 2 8 , 7 3}$ & +1 & 1 & $+3528,73$ & 3517,050 & 11,680 \\
\hline $\mathbf{2 0 1 8}$ & $\mathbf{4 0 7 4 , 3 1}$ & +2 & 4 & $+8148,62$ & 4073,126 & 1,184 \\
\hline $\mathbf{2 0 1 9}$ & $\mathbf{1 4 8 0 4 , 8 7}$ & & 10 & 5560,78 & $\mathbf{1 4 8 0 4 , 8 7 0}$ & 59,364 \\
\hline TOTAL & & & & & & \multicolumn{5}{|c|}{ LINEAR TENDENCY } \\
\hline
\end{tabular}

$$
\begin{gathered}
a=\frac{\sum_{i=1}^{n} \wp_{i} \sum_{i=1}^{n} t_{i}{ }^{2}-\sum_{i=1}^{n} \wp_{i} t_{i} \sum_{i=1}^{n} t_{i}}{n \sum_{i=1}^{n} t_{i}{ }^{2}-\left(\sum_{i=1}^{n} t_{i}\right)^{2}}=\frac{14804,87 \cdot 10}{5 \cdot 10}=2960,974 \\
b=\frac{n \sum_{i=1}^{n} \wp_{i} t_{i}-\sum_{i=1}^{n} t_{i} \sum_{i=1}^{n} \wp_{i}}{n \sum_{i=1}^{n} t_{i}{ }^{2}-\left(\sum_{i=1}^{n} t_{i}\right)^{2}}=\frac{5 \cdot 5560,78}{5 \cdot 10}=556,076 \\
v_{I}=\left[\frac{\sum_{i=1}^{n}\left|\wp_{i}-\wp_{t_{i}}^{I}\right|}{n}: \frac{\sum_{i=1}^{n} \wp_{i}}{n}\right] \cdot 100=\frac{\sum_{i=1}^{n}\left|\wp_{i}-\wp_{t_{i}}^{I}\right|}{\sum_{i=1}^{n} \wp_{i}} \cdot 100=\frac{59,364}{14804,87} \cdot 100=0,4 \%
\end{gathered}
$$

- if the "portfolio of the mathematical operations" for $\wp$ variable, where $\wp=$ the worlwide revenues concerning the marketing of drones, provides a quadratic itinerary $\wp_{t_{i}}=a+b \cdot t_{i}+c t_{i}^{2}, a$ and $b$ will be [5]: 
Table 8 The rank of numbers regarding the the worlwide revenues concerning the merchandising of drones, if this brings forward a quadratic itinerary

\begin{tabular}{|c|c|c|c|c|c|c|c|c|}
\hline \multirow{2}{*}{ YEARS } & \multirow{2}{*}{$\begin{array}{l}\text { THE WORLDWIDE } \\
\text { REVENUES } \\
\text { CONCERNING THE } \\
\text { MERCHANDISING } \\
\text { OF DRONES } \\
\text { (millions \$) } \\
\left(\wp_{i}\right)\end{array}$} & \multicolumn{7}{|c|}{ PARABOLIC TENDENCY } \\
\hline & & $t_{i}$ & $t_{i}^{2}$ & $t_{i}^{3}$ & $t_{i}^{4}$ & $t_{i}^{2} \wp_{i}$ & $\wp_{t_{i}}=a+b t_{i}+c t_{i}^{2}$ & $\mid \wp_{i}-\wp_{t_{i}}$ \\
\hline 2015 & 1865,64 & -2 & 4 & -8 & 16 & 7462,56 & 1857,976295 & 7,66 \\
\hline 2016 & 2385,29 & -1 & 1 & -1 & 1 & 2385,29 & 2400,320857 & 15,03 \\
\hline 2017 & 2950,90 & 0 & 0 & 0 & 0 & 0 & 2951,819714 & 0,92 \\
\hline 2018 & 3528,73 & +1 & 1 & +1 & 1 & 3528,73 & 3512,472857 & 16,26 \\
\hline 2019 & 4074,31 & +2 & 4 & +8 & 16 & 16297,24 & 4082,280285 & 7,97 \\
\hline TOTAL & 14804,87 & & 10 & & 34 & 29673,82 & 14804,87 & 47,84 \\
\hline
\end{tabular}

$$
\begin{gathered}
a=\frac{\sum_{i=1}^{n} t_{i}^{4} \sum_{i=1}^{n} \wp_{i}-\sum_{i=1}^{n} t_{i}^{2} \sum_{i=1}^{n} t_{i}^{2} \cdot \wp_{i}}{n \sum_{i=1}^{n} t_{i}{ }^{4}-\left(\sum_{i=1}^{n} t_{i}^{2}\right)^{2}}=\frac{34 \cdot 14804,87-10 \cdot 29673,82}{5 \cdot 34-10^{2}}=2951,819714 \\
b=\frac{\sum_{i=1}^{n} \wp_{i} t_{i}}{\sum_{i=1}^{n} t_{i}{ }^{2}}=\frac{5560,78}{10}=556,076 \\
c=\frac{n \cdot \sum_{i=1}^{n} t_{i}^{2} \cdot \wp_{i}-\sum_{i=1}^{n} t_{i}^{2} \cdot \sum_{i=1}^{n} \wp_{i}}{n \sum_{i=1}^{n} t_{i}{ }^{4}-\left(\sum_{i=1}^{n} t_{i}^{2}\right)^{2}}=\frac{5 \cdot 29673,82-10 \cdot 14804,87}{5 \cdot 34-10^{2}}=4,577142857 \\
v_{I I}=\left[\frac{\sum_{i=1}^{n} \mid \wp_{i}-\wp_{t_{i}}^{I I}}{n}: \frac{\sum_{i=1}^{n} \wp_{i}}{n}\right] \cdot 100=\frac{\sum_{i=1}^{n}\left|\wp_{i}-\wp_{t_{i}}^{I I}\right|}{\sum_{i=1}^{n} \wp_{i}} \cdot 100=\frac{47,84}{14804,87} \cdot 100=0,32 \%
\end{gathered}
$$

- if the „portfolio of the mathematical operations” for $\wp$ variable, where $\wp=$ the worlwide revenues concerning the marketing of drones, provides a parabolic itinerary of three degree

\begin{tabular}{|c|c|c|c|c|c|c|c|c|c|}
\hline \multirow{2}{*}{ YEARS } & \multirow{2}{*}{$\begin{array}{c}\text { THE } \\
\text { WORLDWIDE } \\
\text { REVENUES } \\
\text { CONCERNING } \\
\text { THE } \\
\text { MERCHANDI- } \\
\text { SING OF } \\
\text { DRONES } \\
\text { (millions \$) } \\
\left(\wp_{i}\right)\end{array}$} & \multicolumn{8}{|c|}{ PARABOLIC TENDENCY OF THREE DEGREE } \\
\hline & & $t_{i}$ & $t_{i}^{2}$ & $t_{i}^{3}$ & $t_{i}^{4}$ & $t_{i}^{6}$ & $t_{i}^{3} \wp_{i}$ & $\wp_{t_{i}}=a+b \cdot t_{i}+c t_{i}^{2}+d t_{i}^{3}$ & $\left|\wp_{i}-\wp_{t_{i}}\right|$ \\
\hline 2015 & 1865,64 & -2 & 4 & -8 & 16 & 64 & $-14925,12$ & 1865,793285 & 0,15 \\
\hline 2016 & 2385,29 & -1 & 1 & -1 & 1 & 1 & $-2385,29$ & 2384,676857 & 0,61 \\
\hline 2017 & 2950,90 & 0 & 0 & 0 & 0 & 0 & 0 & 2951,819714 & 0,92 \\
\hline 2018 & 3528,73 & +1 & 1 & +1 & 1 & 1 & $+3528,73$ & 3528,116857 & 0,61 \\
\hline 2019 & 4074,31 & +2 & 4 & +8 & 16 & 64 & $+32594,48$ & 4074,463285 & 0,15 \\
\hline TOTAL & 14804,87 & & 10 & & 34 & 130 & 18812,8 & 14804,87 & 2,44 \\
\hline
\end{tabular}
$\wp_{t_{i}}=a+b \cdot t_{i}+c t_{i}^{2}+d t_{i}^{3}, a, b, c$ and $d$ will be [5]:

Table 9 The rank of numbers concerning the the worlwide revenues concerning the merchandising of drones, if this brings forward a quadratic itinerary of three degree 


$$
\begin{aligned}
& a=\frac{\sum_{i=1}^{n} t_{i}^{4} \sum_{i=1}^{n} \wp_{i}-\sum_{i=1}^{n} t_{i}^{2} \sum_{i=1}^{n} t_{i}^{2} \cdot \wp_{i}}{n \sum^{n} t_{i}{ }^{4}-\left(\sum^{n} t_{i}^{2}\right)^{2}}=\frac{34 \cdot 14804,87-10 \cdot 29673,82}{5 \cdot 34-10^{2}}=2951,819714 \\
& n \sum_{i=1}^{n} t_{i}^{4}-\left(\sum_{i=1}^{n} t_{i}^{2}\right)^{2} \\
& b=\frac{\sum_{i=1}^{n} t_{i}^{6} \cdot \sum_{i=1}^{n} t_{i} \cdot \wp_{i}-\sum_{i=1}^{n} t_{i}^{4} \cdot \sum_{i=1}^{n} t_{i}^{3} \cdot \wp_{i}}{\sum_{i=1}^{n} t_{i}{ }^{2} \cdot \sum_{i=1}^{n} t_{i}^{6}-\left(\sum_{i=1}^{n} t_{i}^{4}\right)^{2}}=\frac{130 \cdot 5560,78-34 \cdot 18812,8}{10 \cdot 130-34^{2}}=578,2375 \\
& c=\frac{n \cdot \sum_{i=1}^{n} t_{i}^{2} \cdot \wp_{i}-\sum_{i=1}^{n} t_{i}^{2} \cdot \sum_{i=1}^{n} \wp_{i}}{n \sum_{i=1}^{n} t_{i}{ }^{4}-\left(\sum_{i=1}^{n} t_{i}^{2}\right)^{2}}=\frac{5 \cdot 29673,82-10 \cdot 14804,87}{5 \cdot 34-10^{2}}=4,577142857 \\
& d=\frac{\sum_{i=1}^{n} t_{i}^{2} \cdot \sum_{i=1}^{n} t_{i}^{3} \cdot \wp_{i}-\sum_{i=1}^{n} t_{i}^{4} \cdot \sum_{i=1}^{n} t_{i} \cdot \wp_{i}}{\sum_{i=1}^{n} t_{i}^{2} \cdot \sum_{i=1}^{n} t_{i}^{6}-\left(\sum_{i=1}^{n} t_{i}^{4}\right)^{2}}=\frac{10 \cdot 18812,8-34 \cdot 5560,78}{10 \cdot 130-34^{2}}=-6,5175 \\
& v_{I I I}=\left[\frac{\sum_{i=1}^{m}\left|\wp_{i}-\wp_{t_{i}}^{I I I}\right|}{n}: \frac{\sum_{i=1}^{m} \wp_{i}}{n}\right] \cdot 100=\frac{\sum_{i=1}^{m}\left|\wp_{i}-\wp_{t_{i}}^{I I I}\right|}{\sum_{i=1}^{m} \wp_{i}} \cdot 100=\frac{2,44}{14804,87} \cdot 100=0,02 \%
\end{aligned}
$$

\begin{tabular}{|c|c|c|c|c|c|c|c|}
\hline \multirow{2}{*}{ YEARS } & \multirow{2}{*}{$\begin{array}{c}\text { THE WORLDWIDE } \\
\text { REVENUES } \\
\text { CONCERNING THE } \\
\text { MERCHANDISING OF } \\
\text { DRONES } \\
\text { (millions \$) }\left(\wp_{i}\right)\end{array}$} & \multicolumn{6}{|c|}{ EXPONENTIAL TENDENCY } \\
\hline & & $t_{i}$ & $\lg \wp_{i}$ & $t_{i} \lg \wp_{i}$ & $\lg \wp_{t_{i}}=\lg a+t_{i} \lg b$ & $\wp_{t_{i}}=a b^{t_{i}}$ & $\left|\wp_{i}-\wp_{t_{i}}\right|$ \\
\hline 2015 & 1865,64 & -2 & 3,270827845 & $-6,541655689$ & 3,285493307 & 1929,715597 & 64,08 \\
\hline 2016 & 2385,29 & -1 & 3,377541187 & $-3,377541187$ & 3,370346256 & 2346,098576 & 39,19 \\
\hline 2017 & 2950,90 & 0 & 3,469954492 & 0 & 3,455199205 & 2852,326291 & 98,57 \\
\hline 2018 & 3528,73 & +1 & 3,547618430 & $+3,547618430$ & 3,540052154 & 3467,784922 & 60,94 \\
\hline 2019 & 4074,31 & +2 & 3,610054070 & $+7,220108140$ & 3,624905103 & 4216,043693 & 141,73 \\
\hline TOTAL & 14804,87 & & 17,27599602 & 0,848529492 & & & 404,51 \\
\hline
\end{tabular}

- if the "portfolio of the mathematical operations" for $\omega$ variable, where $\wp=$ the worlwide revenues concerning the marketing of drones, provides an exponential itinerary $\wp_{t_{i}}=a b^{t_{i}}, a$ and $b$ will be [5]:

Table 10 The rank of numbers regarding the worlwide revenues concerning the merchandising of drones, if this brings forward an exponential itinerary

$$
\begin{gathered}
\lg a=\frac{\sum_{i=1}^{n} \lg \wp_{i} \sum_{i=1}^{n} t_{i}{ }^{2}-\sum_{i=1}^{n} t_{i} \lg \wp_{i} \sum_{i=1}^{n} t_{i}}{n \sum_{i=1}^{n} t_{i}{ }^{2}-\left(\sum_{i=1}^{n} t_{i}\right)^{2}}=\frac{17,27599602 \cdot 10}{5 \cdot 10}=3,455199205 \\
\lg b=\frac{n \cdot \sum_{i=1}^{n} t_{i} \lg \wp_{i}-\sum_{i=1}^{n} \lg \wp_{i} \sum_{i=1}^{n} t_{i}}{n \sum_{i=1}^{n} t_{i}{ }^{2}-\left(\sum_{i=1}^{n} t_{i}\right)^{2}}=\frac{5 \cdot 0,848529492}{5 \cdot 10}=0,0848529492 \\
v_{\exp }=\left[\frac{\sum_{i=1}^{n}\left|\wp_{i}-\wp_{t_{i}}^{\exp }\right|}{n}: \frac{\sum_{i=1}^{n} \wp_{i}}{n}\right] \cdot 100=\frac{\sum_{i=1}^{n}\left|\wp_{i}-\wp_{t_{i}}^{\exp }\right|}{\sum_{i=1}^{n} \wp_{i}} \cdot 100=\frac{404,51}{14804,87} \cdot 100=2,73 \% \\
v_{I I I}=0,02 \%<v_{I I}=0,32 \%<v_{I}=0,4 \%<v_{\exp }=2,73 \%
\end{gathered}
$$


The "portfolio of the mathematical operations" which focuses the worldwide revenues concerning the marketing of drones, brings forward a parabolic itinerary of three degree $\wp_{t_{i}}=a+b \cdot t_{i}+c t_{i}^{2}+d t_{i}^{3}$

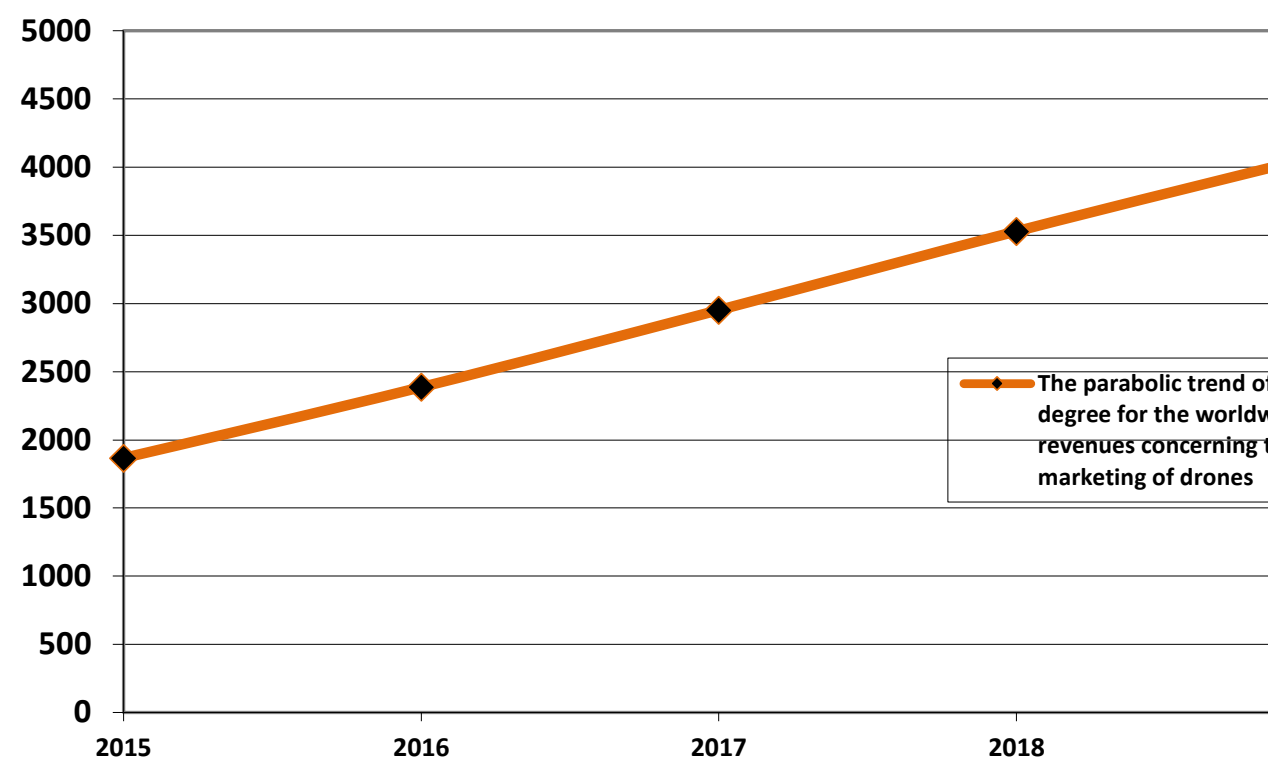

Graph 3 The quadratic itinerary of three degree for the values which stipulate the evolution of the worldwide revenues regarding the marketing of drones

$$
\begin{aligned}
& \omega_{2020}^{\text {MARKETING_OF_DRONES }}=2951,819714+578,2375 \cdot 3+4,577142857 \cdot 3^{2}+(-6,5175) \cdot 3^{3} \cong 4552 \_ \text {Smillions } \\
& \omega_{2021}^{\text {MARKETING_0F_DRONES }}=2951,819714+578,2375 \cdot 4+4,577142857 \cdot 4^{2}+(-6,5175) \cdot 4^{3} \cong 4921 \text { \$millions }
\end{aligned}
$$

\section{Conclusions}

While in 1980, Erick Drexter described the nanotechnology as the engineering which models machines at the molecular scale, robots arms, "tiny" computers comparative to the cells, in our days, we can view another definition concerning the nanotechnology, such as the opinion of the U.S. National Nanotechnology Initiative, which expresses that, whatever entity which is under 100 nanometers, with new properties, represents the nanotechnology. The drones, these amazing small „creatures”, modify our perceptions about the "angles" which offer possibilities to make „insertions" in the worldwide stratosphere. There are a lot of drones on worldwide level: drones which perform the remote zapper for to guide the vehicles, police-drones which supervise if the people respect the restrictions of confinement as effects of the Covid 19 virus, mini drones with the roles of weapons used in the conflict zones, such as: Irak, Afghanistan, Lybia, Ukraine, Syria and which can terrify the enemy. In the immediate horizont 2020-2021, we can see as the worldwide market values regarding the nanotechnology rise in 2020 to $67,74 \$$ billions and in 2021 , these rise to $77,42 \$$ billions. Also, in the same horizont 2020-2021, the worldwide consumptions of drones touch the values of 49 millions in 2020, respectively 66 millions in 2021 and the worldwide revenues concerning the marketing of drones rise to 4552 \$ millions in 2020, respectively 4921 \$ millions in 2021.

\section{References}

1. Foster L. - "Nanotechnology: Science, Innovation and Opportunity ",Prentice HallPublishing House, 2015.

2. Burrows T. - "Robots, Drones and Radar - Electronic Go to War", Learner Publishing House, Minneapolis, 2017.

3. Chandler M. - "Military Drones", The New-York Press Association Publishing House, New-York, 2017.

4. Cheng E. - "Aerial Photography and Videography Using Drones", Peachpit Press Publishing House, San Francisco, 2015.

5. Gauss C.F. - „Disquisitiones Arithmeticae and other papers on number theory”, english translation Springer Publishing House, New York, 1986

6. Grumezescu A.M. - „Nanoarchitectonics in Biomedicine”, Elsevier Publishing House, New York, 2019.

7. Miah A. - „Drones - The Brilliant, the Bad and the Beautiful”, Emerald Publishing House, Bingley, U.K., 2020.

8. Ramsden J.J. - „Nanotechnology: An Introduction”, Elsevier Publishing House, Amsterdam, 2011.

9. Ramsden J.J. - „Applied Nanotechnology”, Elsevier Publishing House, Oxford, 2009.

10. Schuety C. - „The American Way of Swarm. A Machine Learning Strategy for Training Autonomous Systems - UAV and UUV Drone Attack Force Combat Decentralized Execution With Artificial Intelligence”, Department of Defence, U.S. Government, U.S. Military Publishing House, Washington D.C., 2019.

11. Stubblefield T. - „Drone Art - The Everywhere War as Medium”, University of California Press Publishing House, Los Angeles, 2020.

12. Wolf E.L. - „Nanophysics and Nanotechnology: An Introduction to Modern Concepts in Nanoscience”, Wiley Publishing House, NewYork, 2006. 